\title{
Influence of Body Mass Index on Long-Term Outcome in Patients with Rectal Cancer-A Single Centre Experience
}

\author{
Maximilian Kalb, Melanie C. Langheinrich, Susanne Merkel, Christian Krautz, Maximilian Brunner, \\ Alan Bénard, Klaus Weber, Christian Pilarsky D, Robert Grützmann and Georg F. Weber *
} \begin{abstract}
max.kalb@gmx.net (M.K.); melanie.langheinrich@uk-erlangen.de (M.C.L.); susanne.merkel@uk-erlangen.de (S.M.); christian.krautz@uk-erlangen.de (C.K.); maximilian.brunner@uk-erlangen.de (M.B.); alan.benard@uk-erlangen.de (A.B.); klaus.weber@uk-erlangen.de (K.W.); christian.pilarsky@uk-erlangen.de (C.P.); robert.gruetzmann@uk-erlangen.de (R.G.)

* Correspondence: georg.weber@uk-erlangen.de; Tel.: +49-9131-85-33296
\end{abstract}

Department of Surgery, Erlangen University Hospital, Krankenhausstraße 12, 91054 Erlangen, Germany;

Received: 10 April 2019; Accepted: 26 April 2019; Published: 30 April 2019

\begin{abstract}
Background: Excess bodyweight is known to influence the risk of colorectal cancer; however, little evidence exists for the influence of the body mass index (BMI) on the long-term outcome of patients with rectal cancer. Methods: We assessed the impact of the BMI on the risk of local recurrence, distant metastasis and overall—survival in 612 patients between 2003 and 2010 after rectal cancer diagnosis and treatment at the University Hospital Erlangen. A Cox-regression model was used to estimate the hazard ratio and multivariate risk of mortality and distant-metastasis. Median follow up-time was 58 months. Results: Patients with obesity class II or higher (BMI $\left.\geq 35 \mathrm{~kg} / \mathrm{m}^{2}, n=25\right)$ and patients with underweight $\left(\mathrm{BMI}<18.5 \mathrm{~kg} / \mathrm{m}^{2}, n=5\right)$ had reduced overall survival (hazard ratio $(\mathrm{HR})=1.6 ; 95 \%$ confidence interval (CI) 0.9-2.7) as well as higher rates of distant metastases (hazard ratio HR $=1.7$; 95\% CI 0.9-3.3) as compared to patients with normal bodyweight $\left(18.5 \leq \mathrm{BMI}<25 \mathrm{~kg} / \mathrm{m}^{2}, n=209\right)$, overweight $(25 \leq \mathrm{BMI}$ $\left.<30 \mathrm{~kg} / \mathrm{m}^{2}, n=257\right)$ or obesity class I $\left(30 \leq \mathrm{BMI}<35 \mathrm{~kg} / \mathrm{m}^{2}, n=102\right)$. There were no significant differences for local recurrence. Conclusions: Underweight and excess bodyweight are associated with lower overall survival and higher rates of distant metastasis in patients with rectal cancer.
\end{abstract}

Keywords: body mass index; rectal cancer; overall survival; distant metastasis

\section{Introduction}

In 2014 , more than 18,500 people in Germany were initially diagnosed with rectal cancer $(11,414$ males and 7281 females) [1], along with 7605 rectal cancer deaths (4519 in males and 3086 in females) [1]. The 5-year survival rate for rectal cancer increased in the 21st century from $44.3 \%$ in the early nineties (1990 to 1992 ) to $53.7 \%$ in the period of 2000-2002, earlier detection and progress in therapy being the cause [2]. Colorectal cancer is responsible for the third highest economic cost (13.1 Billion $€, 10 \%$ of all cancer costs) in the European Union behind lung (18.8 billion $€, 15 \%$ ) and breast cancer (15.0 billion $€, 12 \%$ ). Regarding health care costs colorectal cancer ( 5.57 billion $€, 11 \%$ of all-cancer related health care costs) was ranked second behind breast cancer ( 6.73 billion $€, 13 \%$ ) followed by prostate cancer (5.43 billion $€, 11 \%$ ) [3].

Obesity is a well-known risk-factor for the development of colorectal cancer [4,5]. Adiposity triggers several systemic and metabolic alterations that can influence carcinogenesis. It has been shown that obesity is related to enhanced levels of leptin in humans, leading to increasing total numbers of cells in different colonic tissues [6]. In addition, leptin was associated with reducing apoptosis in 
various in vitro experiments [6]. These observations suggest that, in some cases, an un-physiological elevation of leptin might become a risk factor for tumor growth.

In a prospective cohort study, performed in the period from 1986 until 1992, the Body Mass Index (BMI) of 47,723 male patients was correlated with the incidence of colorectal cancer or adenoma. Thereby, BMI was identified as a direct risk factor for the development of colorectal cancer independent of physical activity [7]. A meta-analysis of 31 studies performed in 2007 identified a dose-response correlation between BMI and colorectal cancer, showing that an increase of $2 \mathrm{~kg} / \mathrm{m}^{2}$ elevated the risk for colorectal cancer by 7\% [8]. Underweight impaired the early and long-term survival after rectal cancer surgery in a multicenter observational study [9].

However, the effect of the BMI on the long-term survival for patients with rectal carcinoma has not been assessed in detail. The aim of our study was therefore to investigate the influence of BMI on the long-term survival of patients diagnosed with rectal cancer.

\section{Results}

\subsection{Subject Characteristics}

The median follow-up time for 612 patients was 93 (range 2-156) months. The male-to-female ratio was 1.97 to 1 . The mean age was 65 years (range 18-91), there was no significant difference for age $<65$ and $\geq 65$ between the BMI-groups as classified by the World Health Organisation (WHO) or Group 1 and 2. Female patients were more likely to be underweight than male patients, whereas male patients showed higher rates of overweight, obesity class I and obesity class II. Underweight and obese patients had a higher perioperative risk as classified by the American Society of Anaesthesiologists (ASA) scoring (1-4) $(p=0.005)$. An ASA-Score of 3 or 4 was found more often in individuals graded as underweight, obese class I, obese class II and obese class III. Group 2 showed a significantly higher rate of ASA-Score 3 or 4 , compared to Group $1(p=0.004)$. CEA-levels were significantly more often elevated in Group 2 compared to patients in Group $1(p=0.048)$. Furthermore, patients in Group 2 were more likely to receive a multivisceral resection than patients in Group $1(p=0.042)$. Except for this, no significant differences for the tumour or patient characteristics could be demonstrated for Group 1 and 2. Further characteristics of the patients, tumours and the treatment details are shown in Tables 1 and 2.

Table 1. Demographics, tumour and treatment characteristics of 612 patients.

\begin{tabular}{lcc}
\hline Patient Characteristics & Number & Percent \\
\hline Age (years), median (range) & $65(18-91)$ & \\
Male Sex & 406 & 66.3 \\
Female & 206 & 33.7 \\
BMI Groups & & \\
Group 1 BMI 18.5-35 & 580 & 94.8 \\
Group 2: BMI <18.5 \& $\geq 35$ & 32 & 5.2 \\
BMI WHO & & \\
<18.5 underweight & 7 & 1.1 \\
18.5-24.9 normal weight & 212 & 34.6 \\
25.0-29.9 overweight & 266 & 43.5 \\
30.0-34.9 class I obesity & 102 & 16.7 \\
35.0-39.9 class II obesity & 16 & 2.6 \\
$\geq 40.0$ class III obesity & 9 & 1.5 \\
Tumour site & & \\
<6 cm lower third & 211 & 34.5 \\
6-12 cm middle third & 276 & 45.1 \\
12-16 cm upper third & 125 & 20.4 \\
ASA-Score & & \\
ASA 1 \& 2 & 490 & 80.1 \\
ASA 3 \& 4 & 118 & 19.3 \\
Missing & 4 & 0.7 \\
\hline
\end{tabular}


Table 1. Cont.

\begin{tabular}{|c|c|c|}
\hline Patient Characteristics & Number & Percent \\
\hline \multicolumn{3}{|l|}{ Pretherapy CEA level } \\
\hline $\operatorname{normal}(<5 \mathrm{ng} / \mathrm{L})$ & 438 & 71.6 \\
\hline raised(>10 ng/L) & 94 & 15.4 \\
\hline Missing & 80 & 13.1 \\
\hline \multicolumn{3}{|l|}{ Neoadjuvant therapy } \\
\hline neoadjuvant RCT & 320 & 52.3 \\
\hline neoadjuvant RT & 4 & 0.7 \\
\hline neoadjuvant $\mathrm{CT}$ & 1 & 0.2 \\
\hline None & 287 & 46.9 \\
\hline \multicolumn{3}{|l|}{ Elective surgery } \\
\hline Yes & 606 & 99.0 \\
\hline No, Emergency & 6 & 1.0 \\
\hline \multicolumn{3}{|l|}{ Type of surgery } \\
\hline Anterior resection & 77 & 12.6 \\
\hline Low anterior resection & 378 & 61.8 \\
\hline Hartmann procedure & 8 & 1.3 \\
\hline Intersphincteric resection & 50 & 8.2 \\
\hline Abdominoperineal excision & 99 & 16.2 \\
\hline \multicolumn{3}{|l|}{ Multivisceral resection } \\
\hline No & 520 & 85.0 \\
\hline Yes & 92 & 15.0 \\
\hline \multicolumn{3}{|l|}{ Postoperative therapy } \\
\hline None & 70 & 11.4 \\
\hline $\mathrm{CT}$ & 171 & 27.9 \\
\hline RT & 4 & 0.7 \\
\hline $\mathrm{RCT}$ & 56 & 9.2 \\
\hline unknown & 311 & 50.8 \\
\hline \multicolumn{3}{|l|}{ pT-category } \\
\hline pT1 & 66 & 10.8 \\
\hline pT2 & 103 & 16.8 \\
\hline pT3 & 100 & 16.3 \\
\hline pT4 & 18 & 2.9 \\
\hline урт0 & 60 & 9.8 \\
\hline ypT1 & 13 & 2.1 \\
\hline урT2 & 113 & 18.5 \\
\hline урт3 & 120 & 19.6 \\
\hline урT4 & 19 & 3.1 \\
\hline \multicolumn{3}{|l|}{ pN-category } \\
\hline pNo & 217 & 35.5 \\
\hline $\mathrm{pN} 1$ & 46 & 7.5 \\
\hline pN2 & 24 & 3.9 \\
\hline ypN0 & 235 & 38.4 \\
\hline ypN1 & 69 & 11.3 \\
\hline $\mathrm{ypN} 2$ & 21 & 3.4 \\
\hline \multicolumn{3}{|l|}{ UICC-stage } \\
\hline I & 150 & 24.5 \\
\hline II & 67 & 10.9 \\
\hline III & 70 & 11.4 \\
\hline y0 & 55 & 9.0 \\
\hline yI & 101 & 16.5 \\
\hline yII & 79 & 12.9 \\
\hline yIII & 90 & 14.7 \\
\hline
\end{tabular}

ypT: pathological tumour category after chemoradiation, ypN: pathological node category after chemoradiation, BMI: Body Mass Index, WHO: World Health Orga, ASA: American Society of Anaesthesiologists, CEA: Carcinoembryonic antigen, RCT: Radiochemotherapy, CT: Chemotherapy, UICC: Union internatonale contre le cancer. 
Table 2. Demographics, tumour and treatment characteristics of 612 patients for BMI-groups.

\begin{tabular}{|c|c|c|c|c|c|c|c|c|c|c|}
\hline Patient Characteristics & $\begin{array}{l}<18.5 \mathrm{~kg} / \mathrm{m}^{2} \\
\text { Underweight } \\
(n=7)\end{array}$ & $\begin{array}{c}18.5-25 \mathrm{~kg} / \mathrm{m}^{2} \text { Normal } \\
\text { Range }(n=212)\end{array}$ & $\begin{array}{c}25-30 \mathrm{~kg} / \mathrm{m}^{2} \\
\text { Overweight } \\
(n=266)\end{array}$ & $\begin{array}{c}30-35 \mathrm{~kg} / \mathrm{m}^{2} \\
\text { Obese Class I } \\
(n=102)\end{array}$ & $\begin{array}{c}35-40 \mathrm{~kg} / \mathrm{m}^{2} \\
\text { Obese Class } \\
\text { II }(n=16)\end{array}$ & $\begin{array}{c}\geq 40 \mathrm{~kg} / \mathrm{m} 2 \\
\text { Obese Class } \\
\text { III }(n=9)\end{array}$ & $p$ & $\begin{array}{c}\text { Group 1: BMI } \\
18.5-35(n=580)\end{array}$ & $\begin{array}{c}\text { Group 2: BMI } \\
<18.5 \& \geq 35(n=32)\end{array}$ & $p$ \\
\hline \multicolumn{11}{|l|}{ Age } \\
\hline$<65$ & $6(86)$ & $118(55.7)$ & $137(51.5)$ & $45(44.1)$ & $8(50)$ & $6(67)$ & & $300(51.7)$ & $20(63)$ & \\
\hline$\geq 65$ & $1(14)$ & $94(44.3)$ & $129(48.5)$ & $57(55.9)$ & $8(50)$ & $3(33)$ & 0.180 & $280(48.3)$ & $12(37)$ & 0.277 \\
\hline \multicolumn{11}{|l|}{ Sex } \\
\hline Male & $3(43)$ & $122(57.5)$ & $196(73.7)$ & $69(67.6)$ & $11(69)$ & $5(56)$ & & $387(66.7)$ & $19(59)$ & \\
\hline Female & $4(57)$ & $90(42.5)$ & $70(26.3)$ & $33(32.4)$ & $5(31)$ & $4(44)$ & 0.005 & $193(33.3)$ & $13(41)$ & 0.443 \\
\hline \multicolumn{11}{|l|}{ Tumour site } \\
\hline$<6 \mathrm{~cm}$ lower third & $4(57)$ & $83(39.2)$ & $82(30.8)$ & $34(33.3)$ & $5(31)$ & $3(33)$ & & $199(34.3)$ & $12(38)$ & \\
\hline$<12 \mathrm{~cm}$ middle third & $3(43)$ & $84(39.6)$ & $132(49.6)$ & $43(42.2)$ & $9(56)$ & $5(56)$ & & 259 (44.7) & 17 (53) & \\
\hline$\leq 16 \mathrm{~cm}$ upper third & $0(0)$ & $45(21.2)$ & $52(19.6)$ & $25(24.5)$ & $2(13)$ & $1(11)$ & 0.416 & $122(21.0)$ & $3(9)$ & 0.269 \\
\hline ASA $1 \& 2$ & $4(57)$ & 181/211 (85.8) & 215/264 (81.4) & 75/101 (74.3) & $9(56)$ & $6(67)$ & & $471 / 576(81.8)$ & $19(59)$ & \\
\hline ASA $3 \& 4$ & $3(43)$ & $30 / 211$ (14.2) & $49 / 264$ (18.6) & $26 / 101(25.7)$ & $7(44)$ & $3(33)$ & 0.005 & 105/576 (18.2) & $13(41)$ & 0.004 \\
\hline \multicolumn{11}{|l|}{ Pretherapy CEA level $\mathrm{b}$} \\
\hline Normal $(<5 \mathrm{ng} / \mathrm{L})$ & $3 / 6(50)$ & 143/176 (81.3) & 196/231 (84.8) & 78/94 (83.0) & $9(56)$ & $9(100)$ & & $417 / 501(83.2)$ & $21 / 31(68)$ & \\
\hline Elevated $(\geq 5 \mathrm{ng} / \mathrm{L})$ & $3 / 6(50)$ & $33 / 176(18.7)$ & $35 / 231(14.2)$ & $16 / 94(17.0)$ & $7(44)$ & $0(0)$ & 0.018 & $84 / 501(16.8)$ & $10 / 31(32)$ & 0.048 \\
\hline $\begin{array}{l}\text { Neoadjuvant therapy } \\
\text { Neoadjuvant RCT }\end{array}$ & $6(86)$ & $117(55.2)$ & $133(50.0)$ & $52(51.0)$ & $9(56)$ & $9(47)$ & & $302(52.1)$ & $18(56)$ & \\
\hline Neoadjuvant RT & $0(0)$ & $1(0.5)$ & $3(1.1)$ & $0(0.0)$ & $0(0)$ & $0(0)$ & & $4(0.7)$ & $0(0)$ & \\
\hline Neoadjuvant $\mathrm{CT}$ & $0(0)$ & $0(0.0)$ & $1(0.4)$ & $0(0.0)$ & $0(0)$ & $0(0)$ & & $1(0.1)$ & $0(0)$ & \\
\hline Elective surgery & $1(14)$ & $94(44.3)$ & $129(48.5)$ & $50(49.0)$ & $7(44)$ & $10(53)$ & 0.635 & $273(47.1)$ & $14(44)$ & 0.786 \\
\hline Yes & $7(100)$ & $208(98.1)$ & $264(99.2)$ & $102(100)$ & $16(100)$ & $9(100)$ & & $574(99.0)$ & $32(100)$ & \\
\hline & Type of surgery & $4(1.9)$ & $2(0.8)$ & $0(0.0)$ & $0(0)$ & $0(0)$ & 0.523 & $6(1.0)$ & $0(0)$ & 1.0 \\
\hline Anterior resection & $0(0)$ & $32(15.1)$ & $28(10.5)$ & $15(14.7)$ & $1(6)$ & $1(11)$ & & $75(12.9)$ & $2(6)$ & \\
\hline Low anterior resection & $2(29)$ & $126(59.4)$ & $177(66.5)$ & $59(57.8)$ & $9(56)$ & $5(56)$ & & $362(62.4)$ & $16(50)$ & \\
\hline Hartmann procedure & $1(14)$ & $3(1.4)$ & $3(1.1)$ & $1(1.1)$ & $0(0)$ & $0(0)$ & & $7(1.3)$ & $1(3)$ & \\
\hline Intersphincteric resection & $0(0)$ & $15(7.1)$ & $19(7.1)$ & $13(12.7)$ & $2(13)$ & $1(11)$ & & $47(8.1)$ & $3(9)$ & \\
\hline $\begin{array}{l}\text { Abdominoperineal } \\
\text { excision }\end{array}$ & $4(57)$ & $36(17.0)$ & $39(14.8)$ & $14(13.7)$ & $4(25)$ & $2(22)$ & 0.863 & $89(15.3)$ & $10(32)$ & 0.086 \\
\hline \multicolumn{11}{|l|}{ Multivisceral resection } \\
\hline No & $3(43)$ & $181(85.4)$ & $228(85.7)$ & $88(86.3)$ & $13(81)$ & $7(78)$ & & $497(85.7)$ & $23(72)$ & \\
\hline Yes & $4(57)$ & $31(14.6)$ & $38(14.3)$ & $14(13.7)$ & $3(19)$ & $2(22)$ & 0.098 & $83(14.3)$ & $9(28)$ & 0.042 \\
\hline \multicolumn{11}{|l|}{ Postoperative therapy ${ }^{c}$} \\
\hline RCT & $0 / 6(0)$ & 19/105 (18.1) & $26 / 127(20.5)$ & $8 / 49(16.3)$ & 1/9 (11) & $2 / 5(40)$ & & $53 / 281(18.9)$ & 3/20 (15) & \\
\hline Radiotherapy & $0 / 6(0)$ & 0/105 (0.0) & $2 / 127(1.6)$ & $2 / 49(4.2)$ & $0 / 9(0)$ & $0 / 5(0)$ & & 4/281 (1.4) & $0 / 20(0)$ & \\
\hline Chemotherapy & $5 / 6(83.3)$ & $55 / 105$ (52.4) & $77 / 127(60.6)$ & $28 / 49(57.1)$ & $5 / 9(56)$ & $1 / 5(20)$ & & $160 / 281(56.9)$ & $11 / 20(55)$ & \\
\hline None & $1 / 6(16.7)$ & $31 / 105$ (29.5) & 22/127 (17.3) & 11/49. (22.4) & 3/9 (33) & $2 / 5(40)$ & 0.320 & $64 / 281(22.8)$ & 6/20 (30) & 0.856 \\
\hline
\end{tabular}


Table 2. Cont.

\begin{tabular}{|c|c|c|c|c|c|c|c|c|c|c|}
\hline Patient Characteristics & $\begin{array}{c}<18.5 \mathrm{~kg} / \mathrm{m}^{2} \\
\text { Underweight } \\
\quad(n=7)\end{array}$ & $\begin{array}{l}18.5-25 \mathrm{~kg} / \mathrm{m}^{2} \text { Normal } \\
\text { Range }(n=212)\end{array}$ & $\begin{array}{c}25-30 \mathrm{~kg} / \mathrm{m}^{2} \\
\text { Overweight } \\
(n=266)\end{array}$ & $\begin{array}{c}30-35 \mathrm{~kg} / \mathrm{m}^{2} \\
\text { Obese Class I } \\
(n=102)\end{array}$ & $\begin{array}{c}35-40 \mathrm{~kg} / \mathrm{m}^{2} \\
\text { Obese Class } \\
\text { II }(n=16)\end{array}$ & $\begin{array}{c}\geq 40 \mathrm{~kg} / \mathrm{m} 2 \\
\text { Obese Class } \\
\text { III }(n=9)\end{array}$ & $p$ & $\begin{array}{c}\text { Group 1: BMI } \\
\text { 18.5-35 }(n=580)\end{array}$ & $\begin{array}{c}\text { Group 2: BMI } \\
<18.5 \& \geq 35(n=32)\end{array}$ & $p$ \\
\hline \multicolumn{11}{|l|}{ pT-category } \\
\hline pT1 & $0(0)$ & $22(10.4)$ & $25(9.4)$ & $14(13.7)$ & $2(13)$ & $3(34)$ & & $61(10.5)$ & $5(16)$ & \\
\hline pT2 & $0(0)$ & $31(14.6)$ & $49(18.4)$ & $20(19.6)$ & $2(13)$ & $1(11)$ & & $100(17.2)$ & $3(9)$ & \\
\hline pT3 & $1(14)$ & $34(16.0)$ & $45(16.9)$ & $15(14.7)$ & $3(19)$ & $2(22)$ & & $94(16.2)$ & $6(19)$ & \\
\hline pT4 & $0(0)$ & $7(3.3)$ & $10(3.8)$ & $1(1.1)$ & $0(0)$ & $0(0)$ & & $18(3.2)$ & $0(0)$ & \\
\hline урт0 & $0(0)$ & $22(10.4)$ & $29(10.9)$ & $7(6.9)$ & $1(6)$ & $1(11)$ & & $58(10.0)$ & $2(6)$ & \\
\hline урT1 & $0(0)$ & $5(2.4)$ & $7(2.6)$ & $1(1.1)$ & $0(0)$ & $0(0)$ & & $13(2.2)$ & $0(0)$ & \\
\hline урт2 & $1(14)$ & $46(21.7)$ & $45(16.9)$ & $18(17.5)$ & $3(19)$ & $0(0)$ & & $109(18.8)$ & 4 (13) & \\
\hline урт3 & $3(43)$ & $39(18.4)$ & $48(18.0)$ & $24(23.5)$ & $4(24)$ & $2(22)$ & & $111(19.1)$ & $9(28)$ & \\
\hline урт4 & $2(29)$ & $6(2.8)$ & $8(3.1)$ & $2(1.9)$ & $1(6)$ & $0(0)$ & 0.110 & $16(2.8)$ & $3(9)$ & 0.333 \\
\hline \multicolumn{11}{|l|}{ pN-category } \\
\hline pNO & $1(14)$ & $67(31.6)$ & $100(37.6)$ & $42(41.2)$ & $4(25)$ & $3(33)$ & & $209(36.0)$ & $8(25)$ & \\
\hline pN1 & $0(0)$ & $16(7.5)$ & 20 (7.5) & $6(5.9)$ & $2(13)$ & $2(23)$ & & $42(7.2)$ & $4(13)$ & \\
\hline pN2 & $0(0)$ & $11(5.2)$ & $9(3.4)$ & $2(2.0)$ & $1(6)$ & $1(11)$ & & $22(3.8)$ & $2(6)$ & \\
\hline ypNo & $3(43)$ & $90(42.5)$ & $102(38.3)$ & $30(29.4)$ & $7(44)$ & $3(33)$ & & $222(38.3)$ & $13(41)$ & \\
\hline ypN1 & $2(29)$ & $21(9.9)$ & $26(9.8)$ & $19(18.6)$ & $1(6)$ & $0(0)$ & & 66 (11.4) & $3(9)$ & \\
\hline ypN2 & $1(14)$ & $7(3.3)$ & $9(3.4)$ & $3(2.9)$ & $1(6)$ & $0(0)$ & 0.140 & $19(3.3)$ & $2(6)$ & 0.408 \\
\hline
\end{tabular}

a 4 Patients excluded due to unknown ASA-Score, ${ }^{\mathrm{b}} 80$ Patients excluded due to unknown CEA-level, ${ }^{\mathrm{c}} 311$ patients excluded due to missing data on postoperative therapy, Values in parentheses are percentages. 


\subsection{Quality Indicators}

The median number of examined lymph nodes was 21 (range 2-76). Microscopic curative tumor resection rates were significantly lower in patients who were underweight $(p=0.031)$, without significant differences between patients in Group 1 and $2(p=0.238)$. Other than that, there was no discernible difference concerning the surgical quality indicators, showing no significance for aboral $(p=0.474)$ and circumferential $(p=0.445)$ resection margin or intraoperative local tumor cell dissemination $(p=0.186)$. The specimen quality was considered to be good in 555 patients $(97.2 \%)$ without a significant dependency on BMI (WHO, $p=0.309$; Group1 and 2, $p=0.203$ ). The number of abdominoperineal excision (APE) was performed more often in underweight, obese class II and obese class III patients compared to normal weight, overweight, and obese class I patients, but did not reach significance $(p=0.074)$. Group 1 showed a lower rate of APR compared to Group 2 (15.3 vs. $31 \%, p=0.025)$. A primary anastomosis was conducted in $82.5 \%$ with lower count in underweight, obese class II and obese class III individuals $(p=0.019)$ without reaching significant differences for anastomotic leakage ( $p=0.466,4.3 \%$ overall). General postoperative morbidity was increased in underweight, obese class II and obese class III patients compared to normal weight, overweight and obese class I individuals $(p=0.008)$. Surgical complications were significantly increased in the same patient collective $(p=0.006)$, whereas non-surgical complications showed no differences for BMI $(p=0.460)$. The postoperative 30-day mortality was $1.0 \%$ and did not depend on BMI $(p=0.116)$. Further data on quality indicators is shown in Table 3. 
Table 3. Surgical quality indicators for 612 patients.

\begin{tabular}{|c|c|c|c|c|c|c|c|c|c|c|}
\hline Quality Indicators & $\begin{array}{c}<18.5 \\
\text { Underweight } \\
n=7)\end{array}$ & $\begin{array}{l}\text { 18.5-25 Normal } \\
\text { Weight }(n=212)\end{array}$ & $\begin{array}{c}25-30 \\
\text { Overweight } \\
(n=266)\end{array}$ & $\begin{array}{c}\text { 30-35 Obese } \\
\text { Class I } \\
(n=102)\end{array}$ & $\begin{array}{c}\text { 35-40 Obese } \\
\text { Class II } \\
(n=16)\end{array}$ & $\begin{array}{c}\geq 40 \text { Obese } \\
\text { Class III } \\
(n=9)\end{array}$ & $p$ & $\begin{array}{l}\text { Group 1: 18.5-35 } \\
\quad(n=580)\end{array}$ & $\begin{array}{c}\text { Group 2: }<18.5 \& \geq \\
35(n=32)\end{array}$ & $p$ \\
\hline $\begin{array}{l}\text { Number of examined LN; R0, R1, } \\
\text { R2 } n=612^{\mathrm{a}} \text {, median (range) }\end{array}$ & $13(11-33)$ & $20(2-57)$ & $21.5(3-62)$ & $21(5-76)$ & $23.5(8-57)$ & $30(15-43)$ & & $21(2-76)$ & $25.5(8-57)$ & \\
\hline$\geq 12$ LN examined & 6/7 (86) & 188/212 (88.7) & 239/266 (89.8) & 93/102 (91.2) & $15 / 16(94)$ & 9/9 (100) & 0.828 & $520 / 580(89.7)$ & $30 / 32(94)$ & 0.742 \\
\hline pNo $n=217$, median (range) & $33(33-33)$ & $24(12-51)$ & $27(7-57)$ & $31(10-76)$ & $30(22-36)$ & $26(15-41)$ & & $26(7-76)$ & $30(15-41)$ & \\
\hline$\geq 12 \mathrm{LN}$ examined & $1 / 1(100)$ & $67 / 67(100)$ & 98/100 (98.0) & $41 / 42(97.6)$ & $4 / 4(100)$ & $3 / 3(100)$ & 0.664 & 206/209 (98.6) & $8 / 8(100)$ & 0.737 \\
\hline ypNo $n=235$, median (range) & $13(12-13)$ & $17(2-36)$ & $18(3-62)$ & $17(7-37)$ & $21(8-29)$ & $41(16-43)$ & & $17.5(2-62)$ & $16(8-43)$ & \\
\hline$\geq 12 \mathrm{LN}$ examined & $3 / 3(100)$ & $70 / 90(77.8)$ & $79 / 102(77.5)$ & $26 / 30(86.7)$ & $6 / 7(86)$ & $3 / 3(100)$ & 0.004 & $175 / 222(78.8)$ & $12 / 13(92)$ & 0.238 \\
\hline R0-resection-rate & $5 / 7(71)$ & 209/212 (98.6) & $257 / 266(96.6)$ & 102/102 (100) & $16 / 16(100)$ & 9/9 (100) & 0.031 & $568 / 580(97.9)$ & $30 / 32(94)$ & 0.163 \\
\hline Aboral resection margin $>1 \mathrm{~mm}$ & $7 / 7(100)$ & 210/212 (99.1) & $263 / 266(98.9)$ & $100 / 102(98.0)$ & $15 / 16(94)$ & 9/9 (100) & 0.474 & $573 / 580(98.8)$ & $31 / 32(97)$ & 0.351 \\
\hline $\begin{array}{l}\text { Circumferential resection margin > } \\
\qquad 1 \mathrm{~mm}\end{array}$ & $5 / 7(71)$ & 197/212 (92.9) & 244/266 (91.7) & 98/102 (96.1) & $15 / 16(94)$ & 9/9 (100) & 0.186 & $539 / 580(92.9)$ & 29/32 (91) & 0.096 \\
\hline $\begin{array}{l}\text { Intraoperative local tumour cell } \\
\text { dissemination }\end{array}$ & $2 / 7(29)$ & $10 / 212(4.7)$ & $14 / 266(5.3)$ & $2 / 102(2.0)$ & $1 / 16(6)$ & $0 / 9(0)$ & 0.112 & $26 / 580(4.5)$ & $3 / 32(9)$ & 0.279 \\
\hline $\begin{array}{l}\text { Quality of TME/PME }{ }^{\mathrm{b}} \\
\text { Mesorectal/intramesorectal plane }\end{array}$ & $6 / 6(100)$ & 190/196 (96.9) & $246 / 253(97.2)$ & 91/92 (98.9) & $14 / 15(93,3)$ & $8 / 9(88,9)$ & & $527 / 541(97.4)$ & $28 / 30(93)$ & \\
\hline $\begin{array}{l}\text { Muscularis propria plane } \\
\text { Abdominoperineal excision }\end{array}$ & $0 / 6(0)$ & 6/196 (3.1) & $7 / 253(2.8)$ & $1 / 92(1.1)$ & $1 / 15(6,7))$ & $1 / 9(11,1)$ & 0.309 & $14 / 541(2.6)$ & $2 / 30(7)$ & 0.203 \\
\hline All locations & $4 / 7(57)$ & $36 / 212(17.0)$ & $39 / 266(14.7)$ & 14/102 (13.7) & $4 / 16(25)$ & $2 / 9(23)$ & 0.074 & $89 / 580(15.3)$ & $10 / 32(31)$ & 0.025 \\
\hline$<6 \mathrm{~cm}$ lower third & $4 / 4(100)$ & $30 / 83(36.1)$ & $30 / 82(36.6)$ & $14 / 34(41.2)$ & $4 / 5(80)$ & $2 / 3(67)$ & $<0.001$ & $74 / 199(37.2)$ & $10 / 12(83)$ & $<0.001$ \\
\hline 6-12 cm middle third & $0 / 3(0)$ & 6/84 (7.1) & $9 / 132(6.8)$ & $0 / 43(0)$ & $0 / 9(0)$ & $0 / 5(0)$ & $<0.001$ & $15 / 259(5.8)$ & $0 / 17(0)$ & $<0.001$ \\
\hline Anastomosis & $2 / 7(29)$ & $173 / 212(81.6)$ & $224 / 266(84.2)$ & $87 / 102(85.3)$ & $12 / 16(75)$ & 7/9 (78) & 0.019 & $484 / 580(83.4)$ & $21 / 32(66)$ & 0.016 \\
\hline $\begin{array}{l}\text { Anastomotic leak } \\
\text { Morbidity }\end{array}$ & $0 / 2(0)$ & $5 / 173(2.9)$ & $10 / 224(4.5)$ & $7 / 87(8)$ & $0 / 12(0)$ & $0 / 7(0)$ & 0.466 & $22 / 484(4.5)$ & $0 / 21(0)$ & 1.0 \\
\hline Total & 2/7 (29) & 39/212 (18.4) & $55 / 266(20.7)$ & 29/102 (28.4) & $9 / 16(56)$ & 3/9 (33) & 0.008 & $123 / 580(21.2)$ & $14 / 32(44)$ & 0.005 \\
\hline Non-surgical & $0 / 7(0)$, & $9 / 212(4.2)$ & $12 / 266(4.5)$ & $7 / 102(6.8)$ & $1 / 16(6)$ & $0 / 9(0)$ & 0.460 & $26 / 580(4.5)$ & $1 / 32(3)$ & 1.0 \\
\hline Surgical & $2 / 7(29)$ & $30 / 212(14.1)$ & $43 / 266(16.2)$ & 22/102 (21.6) & $8 / 16(50)$ & $3 / 9(33)$ & 0.006 & 95/580 (16.4) & $13 / 32(41)$ & 0.001 \\
\hline Postoperative 30-day mortality & $1 / 7$ (14) & $3 / 212(1.4)$ & $1 / 266(0.4)$ & $1 / 102(1.0)$ & $0 / 16(0)$ & $0 / 9(0)$ & 0.116 & $5 / 580(0.9)$ & $1 / 32(3)$ & 0.282 \\
\hline
\end{tabular}

${ }^{\mathrm{a}} \mathrm{R} 0=598$ patients $+\mathrm{R} 1=9$ patients $+\mathrm{R} 2=5$ patients $=612$ patients; ${ }^{\mathrm{b}} 41$ patients missing due to unknown data. 


\subsection{Local Recurrence}

The 5-year rate for local recurrence in the entire study group was 5.9\% (95\% CI 3.9-7.9). Neither localisation of the tumour nor type of surgery or CEA-level had a significant influence on the 5-year local recurrence. Obesity class II and the presence of perioperative risk factors (ASA 3 and 4) showed a significant increment for local recurrence (Table 4).

Table 4. 5-year rate of locoregional recurrence for 598 patients.

\begin{tabular}{|c|c|c|c|c|c|c|c|}
\hline \multicolumn{5}{|c|}{ Univariate Analysis } & \multicolumn{3}{|c|}{ Multivariate Analysis } \\
\hline $\begin{array}{l}\text { Patient and Tumour } \\
\text { Characteristics }\end{array}$ & Number & $\begin{array}{l}\text { 5Y-locoregional } \\
\text { Recurrence }\end{array}$ & $95 \%$ CI & $p$ & $\begin{array}{c}\text { Hazard } \\
\text { Ratio }\end{array}$ & $95 \%$ CI & $p$ \\
\hline $\begin{array}{c}\text { Total } \\
\text { BI }\end{array}$ & 598 & $5.9 \%$ & $3.9-7.9$ & & & & \\
\hline $\mathrm{BMI}<18.5 \mathrm{~kg} / \mathrm{m}^{2}$ underweight & 5 & $0 \%$ & & & 0.0 & & \\
\hline BMI $18.5-25 \mathrm{~kg} / \mathrm{m}^{2}$ normal range & 209 & $3.7 \%$ & $1.0-6.4$ & & 1.0 & & \\
\hline BMI $25-30 \mathrm{~kg} / \mathrm{m}^{2}$ overweight & 257 & $8.7 \%$ & $5.2-12.2$ & & 1.7 & $0.8-3.7$ & 0.150 \\
\hline BMI $30-35 \mathrm{~kg} / \mathrm{m}^{2}$ obese class I & 102 & $1.1 \%$ & $0.0-3.3$ & & 0.6 & $0.2-1.9$ & 0.357 \\
\hline BMI $35-40 \mathrm{~kg} / \mathrm{m}^{2}$ obese class II & 16 & $23.6 \%$ & $0.0-47.3$ & & 4.1 & $1.1-15.4$ & 0.036 \\
\hline $\begin{array}{c}\text { BMI } \geq 40 \mathrm{~kg} / \mathrm{m}^{2} \text { obese class III } \\
\text { BMI Groups }\end{array}$ & 9 & $11.1 \%$ & $0.0-31.7$ & 0.112 & 2.0 & $0.2-16.3$ & 0.533 \\
\hline Group 1: BMI 18.5-35 & 568 & $5.5 \%$ & $3.5-7.5$ & & 1.0 & & \\
\hline $\begin{array}{c}\text { Group 2: } \mathrm{BMI}<18.5 \& \geq 35 \\
\text { Age }\end{array}$ & 30 & $15.4 \%$ & $1.5-29.3$ & 0.106 & 2.0 & $0.7-5.9$ & 0.187 \\
\hline$\leq 65$ & 315 & $5.6 \%$ & $3.1-8.1$ & & & & \\
\hline$>65$ & 283 & $6.4 \%$ & $3.5-9.3$ & 0.884 & & & \\
\hline Sex & & & & & & & \\
\hline Male & 394 & $6.7 \%$ & $4.2-9.2$ & & & & \\
\hline Female & 204 & $4.3 \%$ & $1.4-7.2$ & 0.111 & & & \\
\hline Localisation & & & & & & & \\
\hline$<6 \mathrm{~cm}$ & 202 & $5.3 \%$ & $2.2-8.4$ & & & & \\
\hline $6-12 \mathrm{~cm}$ & 271 & $7.1 \%$ & $4.0-10.2$ & & & & \\
\hline $12-16 \mathrm{~cm}$ & 125 & $4.3 \%$ & $0.6-8.0$ & 0.641 & & & \\
\hline UICC-stage & & & & & & & \\
\hline 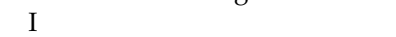 & 150 & $4.3 \%$ & $1.0-7.6$ & & 1.0 & & \\
\hline II & 65 & $11.4 \%$ & $3.4-19.4$ & & 3.8 & $1.4-10.0$ & 0.007 \\
\hline III & 65 & $9.2 \%$ & $1.4-17.0$ & & 1.9 & $0.6-6.1$ & 0.258 \\
\hline y0 & 55 & $0 \%$ & & & 0 & & 0.973 \\
\hline yI & 101 & $3.0 \%$ & $0-6.3$ & & 0.6 & $0.2-2.4$ & 0.487 \\
\hline yII & 74 & $7.5 \%$ & $1.0-14.0$ & & 1.7 & $0.6-5.2$ & 0.321 \\
\hline yIII & 88 & $8.9 \%$ & $2.6-15.2$ & 0.003 & 2.5 & $0.9-6.8$ & 0.067 \\
\hline Abdominoperineal excision & & & & & & & \\
\hline No & 509 & $5.4 \%$ & $3.4-7.4$ & & & & \\
\hline Yes & 89 & $8.9 \%$ & $2.6-15.2$ & 0.104 & & & \\
\hline ASA-Score ${ }^{a}$ & & & & & & & \\
\hline ASA $1 \& 2$ & 481 & $5.0 \%$ & $3.0-7.0$ & & 1.0 & & \\
\hline ASA $3 \& 4$ & 113 & $10.6 \%$ & $4.3-16.9$ & 0.040 & 2.1 & $1.0-4.5$ & 0.042 \\
\hline CEA-level ${ }^{b}$ & & & & & & & \\
\hline Normal (<5 ng/L) & 432 & $5.9 \%$ & $3.5-8.3$ & & 1.0 & & \\
\hline Elevated ( $\geq 5 \mathrm{ng} / \mathrm{L})$ & 88 & $4.9 \%$ & $0.2-9.6$ & 0.389 & 1.9 & $0.9-3.9$ & 0.075 \\
\hline
\end{tabular}

\subsection{Distant Metastasis}

Distant metastases occurred in $16.7 \%$ (95\% CI 13.6-19.8) of all patients in a 5-year observational period. Patients in Group 2 showed a rate of metastatic disease in 31.4\% (95\% CI 14.3-48.5) of cases while patients in Group 1 showed a significantly lower rate of 15.9\% (95 CI 12.8-19.0; $p=0.034$ ) (Figure 1). Significant differences between the BMI Groups as defined by the WHO did not occur. 


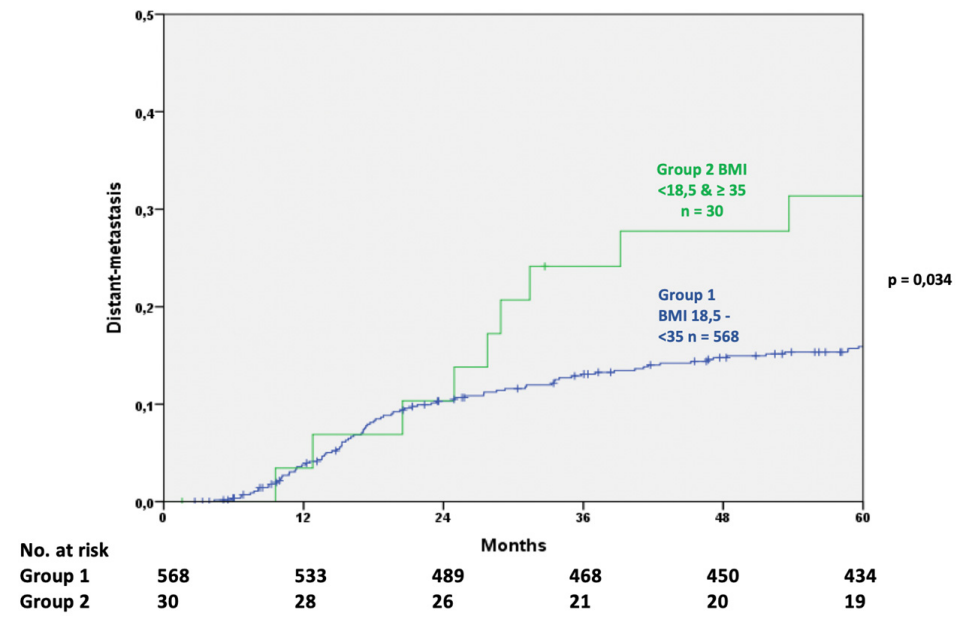

Figure 1. Comparison of distant metastasis after oncological treatment (log rank test).

Type of surgery, the preoperative ASA-Score and localisation of the tumour had no significant influence. Individuals with preoperative CEA levels over $5 \mathrm{ng} / \mathrm{L}$ had a significantly higher rate of distant metastases and an increased risk to develop distant metastases (Table 5).

Table 5. 5-year rate of distant metastases of 598 patients.

\begin{tabular}{|c|c|c|c|c|c|c|c|}
\hline \multicolumn{5}{|c|}{ Univariate Analysis } & \multicolumn{3}{|c|}{ Multivariate Analysis } \\
\hline $\begin{array}{l}\text { Patient and Tumour } \\
\text { Characteristics }\end{array}$ & Number & $\begin{array}{l}\text { 5Y distant } \\
\text { Metastases }\end{array}$ & $95 \%$ CI & $p$ & $\begin{array}{c}\text { Hazard } \\
\text { Ratio }\end{array}$ & $95 \%$ CI & $p$ \\
\hline $\begin{array}{c}\text { Total } \\
\text { BMI WHO }\end{array}$ & \multicolumn{6}{|c|}{ BMI WHO } & \\
\hline $\mathrm{BMI}<18.5 \mathrm{~kg} / \mathrm{m}^{2}$ underweight & 5 & $40.0 \%$ & $0-82.9$ & & 1.7 & $0.4-7.2$ & 0.493 \\
\hline BMI $18.5-25 \mathrm{~kg} / \mathrm{m}^{2}$ normal range & 209 & $16.2 \%$ & $11.1-21.3$ & & 1.0 & & \\
\hline BMI $25-30 \mathrm{~kg} / \mathrm{m}^{2}$ overweight & 257 & $17.3 \%$ & $12.6-22.0$ & & 1.2 & $0.8-2.0$ & 0.340 \\
\hline BMI $30-35 \mathrm{~kg} / \mathrm{m}^{2}$ obese class I & 102 & $11.9 \%$ & $5.6-18.2$ & & 0.9 & $0.5-1.7$ & 0.813 \\
\hline BMI $35-40 \mathrm{~kg} / \mathrm{m}^{2}$ obese class II & 16 & $34.0 \%$ & $9.7-58.3$ & & 1.9 & $0.8-4.8$ & 0.153 \\
\hline $\begin{array}{c}\text { BMI } \geq 40 \mathrm{~kg} / \mathrm{m}^{2} \text { obese class III } \\
\text { BMI Groups }\end{array}$ & 9 & $22.2 \%$ & $0-49.4$ & 0.225 & 1.4 & $0.3-5.8$ & 0.678 \\
\hline Group 1: BMI 18.5-35 & 568 & $15.9 \%$ & $12.8-19.0$ & & 1.0 & & \\
\hline \multicolumn{8}{|l|}{ Age } \\
\hline$\leq 65$ & 315 & $14.1 \%$ & $10.2-18.0$ & & & & \\
\hline$>65$ & 283 & $19.7 \%$ & $14.8-24.6$ & 0.085 & & & \\
\hline \multicolumn{8}{|l|}{ Sex } \\
\hline Male & 394 & $16.3 \%$ & $12.6-20.0$ & & & & \\
\hline Female & 204 & $17.4 \%$ & $12.1-22.7$ & 0.861 & & & \\
\hline \multicolumn{8}{|l|}{ Localisation } \\
\hline$<6 \mathrm{~cm}$ & 202 & $13.8 \%$ & $9.0-18.8$ & & & & \\
\hline $6-12 \mathrm{~cm}$ & 271 & $17.9 \%$ & $13.2-22.6$ & & & & \\
\hline $12-16 \mathrm{~cm}$ & 125 & $18.8 \%$ & $11.7-25.9$ & 0.541 & & & \\
\hline \multicolumn{8}{|l|}{ UICC-stage } \\
\hline 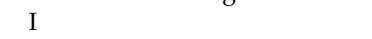 & 150 & $9.8 \%$ & $4.9-14.7$ & & 1.0 & & \\
\hline II & 65 & $23.1 \%$ & $12.5-33.7$ & & 2.1 & $1.0-4.4$ & 0.041 \\
\hline III & 65 & $30.7 \%$ & $18.4-43.0$ & & 2.7 & $1.4-5.5$ & 0.003 \\
\hline y0 & 55 & $1.9 \%$ & $0-5.4$ & & 0.2 & $0-1.3$ & 0.086 \\
\hline yI & 101 & $6.9 \%$ & $2.0-11.8$ & & 0.6 & $0.2-1.4$ & 0.250 \\
\hline yII & 74 & $23.3 \%$ & $13.7-32.9$ & & 2.3 & $1.1-4.6$ & 0.019 \\
\hline yIII & 88 & $30.3 \%$ & $20.5-40.1$ & $<0.001$ & 3.1 & $1.7-5.8$ & $<0.001$ \\
\hline \multicolumn{8}{|l|}{ Abdominoperineal excision } \\
\hline No & 509 & $16.4 \%$ & $13.1-19.7$ & & & & \\
\hline Yes & 89 & $18.0 \%$ & $9.8-26.2$ & 0.313 & & & \\
\hline \multicolumn{8}{|l|}{ ASA-Score ${ }^{a}$} \\
\hline ASA $1 \& 2$ & 481 & $15.2 \%$ & $11.9-18.5$ & & & & \\
\hline ASA $3 \& 4$ & 113 & $24.2 \%$ & $15.8-32.6$ & 0.052 & & & \\
\hline \multicolumn{8}{|l|}{ CEA-level ${ }^{b}$} \\
\hline Normal $(<5 \mathrm{ng} / \mathrm{L})$ & 432 & $14.1 \%$ & $10.8-17.4$ & & 1.0 & & \\
\hline Elevated $(\geq 5 \mathrm{ng} / \mathrm{L})$ & 88 & $31.6 \%$ & $21.6-41.6$ & $<0.001$ & 1.9 & $1.2-3.0$ & 0.005 \\
\hline
\end{tabular}

a 4 Patients excluded due to unknown ASA-Score, ${ }^{\mathrm{b}} 78$ Patients excluded due to unknown CEA-level. 


\subsection{Overall Survival}

The 5-year overall survival was analyzed for 598 patients with an overall survival rate of $82.3 \%$ (CI 79.2-85.4). Patients in Group 1 (BMI $18.5-35 \mathrm{~kg} / \mathrm{m}^{2}$ ) showed an overall survival of $82.9 \%(95 \% \mathrm{CI}$ $79.8-86.0)$, which was significantly higher than in Group $2\left(<18.5\right.$ or $\left.\geq 35 \mathrm{~kg} / \mathrm{m}^{2}\right)$ with $70.0 \%(95 \%$ CI $53.5-86.5 ; p=0.024$ ) (Figure 2). No difference was observed between the BMI Groups as suggested by the WHO.

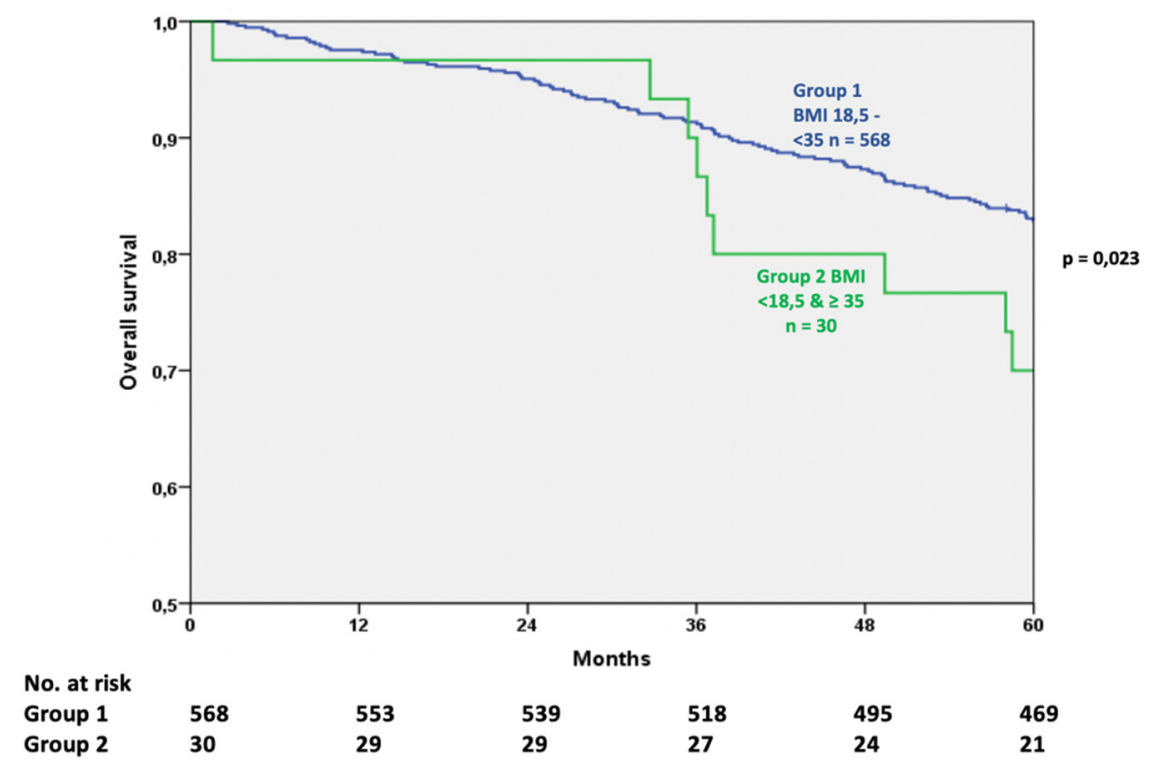

Figure 2. Comparison of overall survival after oncological treatment (log rank test).

The localisation of the tumour had no significant impact, however APE was associated with a significantly lower survival rate. The presence of systemic disabilities, as classified by the ASA-Score, influenced the overall survival. Patients with an ASA-Score of 3 or 4 had a significantly lower 5-year-survial in this period of time. Elevated preoperative CEA-levels were also related to a lower 5-year survival and an increased mortality risk (Table 6). 
Table 6. 5-year overall survival for 598 patients.

\begin{tabular}{|c|c|c|c|c|c|c|c|}
\hline \multicolumn{5}{|c|}{ Univariate Analysis } & \multicolumn{3}{|c|}{ Multivariate Analysis } \\
\hline $\begin{array}{l}\text { Patient and Tumour } \\
\text { Characteristics }\end{array}$ & Number & 5-OSR & $95 \% \mathrm{CI}$ & $p$ & $\begin{array}{c}\text { Hazard } \\
\text { Ratio }\end{array}$ & $95 \% \mathrm{CI}$ & $p$ \\
\hline $\begin{array}{c}\text { Total } \\
\text { BMI WHO }\end{array}$ & 598 & $82.3 \%$ & $79.2-85.4$ & & & & \\
\hline $\mathrm{BMI}<18.5 \mathrm{~kg} / \mathrm{m}^{2}$ underweight & 5 & $60.0 \%$ & $17.1-100$ & & 1.7 & $0.4-7.2$ & 0.458 \\
\hline $\begin{array}{l}\text { BMI } 18.5-25 \mathrm{~kg} / \mathrm{m}^{2} \text { normal } \\
\text { range }\end{array}$ & 209 & $82.3 \%$ & $77.2-87.4$ & & 1.0 & & \\
\hline BMI $25-30$ kg/m² overweight & 257 & $81.3 \%$ & $76.6-86.0$ & & 1.2 & $0.8-1.7$ & 0.369 \\
\hline BMI $30-35 \mathrm{~kg} / \mathrm{m}^{2}$ obese class I & 102 & $88.2 \%$ & 81.9-94.5 & & 0.7 & $0.5-1.2$ & 0.219 \\
\hline BMI $35-40 \mathrm{~kg} / \mathrm{m}^{2}$ obese class II & 16 & $68.8 \%$ & $46.1-91.5$ & & 1.4 & $0.7-2.9$ & 0.377 \\
\hline $\begin{array}{c}\text { BMI } \geq 40 \mathrm{~kg} / \mathrm{m}^{2} \text { obese class III } \\
\text { BMI Groups }\end{array}$ & 9 & $77.8 \%$ & $50.6-100$ & 0.211 & 0.7 & $0.2-2.0$ & 0.524 \\
\hline Group 1: BMI 18.5-35 & 568 & $82.9 \%$ & $79.8-86.0$ & & 1.0 & & \\
\hline $\begin{array}{c}\text { Group 2: } \mathrm{BMI}<18.5 \text { or } \geq 35 \\
\text { Age }\end{array}$ & 30 & $70.0 \%$ & $53.5-86.5$ & 0.023 & 1.1 & $0.7-1.9$ & 0.652 \\
\hline$\leq 65$ & 315 & $90.8 \%$ & $87.7-93.9$ & & & & \\
\hline$>65$ & 283 & $72.8 \%$ & $67.7-77.9$ & $<0.001$ & & & $<0.001$ \\
\hline Sex & & & & & & & \\
\hline Male & 394 & $83.5 \%$ & $79.8-87.2$ & & & & \\
\hline Female & 204 & $79.9 \%$ & $74.4-85.4$ & 0.471 & & & \\
\hline Morbidity & & & & & & & \\
\hline Yes & 132 & $76.5 \%$ & $69.2-83.8$ & & & & \\
\hline No & 466 & $83.9 \%$ & $80.6-87.2$ & 0.004 & & & \\
\hline Localisation & & & & & & & \\
\hline$<6 \mathrm{~cm}$ & 202 & $85.1 \%$ & $80.2-90.0$ & & & & \\
\hline $6-<12 \mathrm{~cm}$ & 271 & $80.4 \%$ & $75.7-85.1$ & & & & \\
\hline $12-16 \mathrm{~cm}$ & 125 & $81.6 \%$ & $74.7-88.5$ & 0.176 & & & \\
\hline UICC-stage & & & & & & & \\
\hline I & 150 & $84.7 \%$ & $79.0-90.4$ & & 1.0 & & \\
\hline II & 65 & $73.8 \%$ & $63.0-84.6$ & & 1.2 & $0.7-2.0$ & 0.476 \\
\hline III & 65 & $63.1 \%$ & $51.3-74.9$ & & 2.3 & $1.5-3.6$ & $<0.001$ \\
\hline y0 & 55 & $98.2 \%$ & $94.7-100$ & & 0.4 & $0.2-0.9$ & 0.024 \\
\hline yI & 101 & $97.0 \%$ & $93.7-100$ & & 0.5 & $0.3-0.8$ & 0.010 \\
\hline yII & 74 & $74.3 \%$ & $64.3-84.3$ & & 1.0 & $0.6-1.7$ & 0.973 \\
\hline yIII & 88 & $78.4 \%$ & $69.8-87.0$ & $<0.001$ & 1.1 & $0.7-1.8$ & 0.658 \\
\hline Abdominoperineal excision & & & & & & & \\
\hline No & 509 & $83.9 \%$ & $80.8-87.0$ & & 1.0 & & \\
\hline Yes & 89 & $73.0 \%$ & $63.8-82.2$ & 0.001 & 1.9 & $1.3-2.9$ & 0.001 \\
\hline ASA-Score ${ }^{a}$ & & & & & & & \\
\hline ASA $1 \& 2$ & 481 & $86.3 \%$ & $83.2-89.4$ & & 1.0 & & \\
\hline $\begin{array}{l}\text { ASA } 3 \& 4 \\
\quad \text { Pretherapy CEA level }^{\mathrm{b}}\end{array}$ & 113 & $64.6 \%$ & $55.8-73.4$ & $<0.001$ & 3.2 & $2.3-4.4$ & $<0.001$ \\
\hline Normal $(<5 \mathrm{ng} / \mathrm{mL})$ & 432 & $84.3 \%$ & $80.8-87.8$ & & 1.0 & & \\
\hline Elevated ( $\geq 5 \mathrm{ng} / \mathrm{mL})$ & 88 & $68.2 \%$ & $58.4-78.0$ & $<0.001$ & 1.9 & $1.3-2.6$ & $<0.001$ \\
\hline
\end{tabular}

\section{Discussion}

In the past years BMI has been proven to be a significant risk factor, or at least a surrogate parameter, for the development of colorectal cancer by different studies [8,10-12]. However, the evidence for BMI being a predictor of outcome in patients with rectal cancer undergoing surgical therapy is still discussed controversially.

Various trials have shown that underweight or obesity are risk factors for reduced survival in colorectal cancer diseases [13-18]. Therefor we decided to search for an indicator in these patients by dividing our patient cohort into two groups (Group 1 BMI 18.5-35 kg/m²; Group 2 BMI $<18.5$ and $\geq 35 \mathrm{~kg} / \mathrm{m}^{2}$ ).

\subsection{Quality Indicators}

We detected differences in the rate of abdominoperineal excision (APE) between tumors in the low and middle third $(45.6 \%$ vs. $3.9 \%$, $p$-value $<0.001)$ of the rectum, but we were not able to confirm the disparate use of APE by sex, with a decreased rate among female patients with rectal cancer, as 
reported by a previous survey [17]. Patients included in our study, treated by APE, had a reduced survival compared to those treated by other surgical procedures (APE vs. other procedures: $73.0 \%$ vs. 83.9\%; HR 2.0, $p$-value < 0.001), however, we did not see differences for distant metastases $(18.0 \%$ vs. $16.4 \%, p$-value 0.313$)$ or local recurrence $(8.9 \%$ vs. $5.4 \%$, $p$-value 0.104$)$. These findings are concordant to a study on 1598 patients with low and mid rectal cancer treated in 38 hospitals being part of the Spanish Rectal Cancer Project [18]. One possible hypothesis is that different patient characteristics, rather than the surgical procedure (APE) itself, cause a difference in oncological outcome in patients undergoing APE [19].

Postoperative complications were seen in $22.2 \%$ of all patients with a significantly higher percentage in underweight and obese class II and III patients. Previous studies have shown that morbidity influences the overall survival (OS) in patients with various tumor diseases [20-22]. We were able to confirm these results in our study (OS Morbidity vs. no Morbidity: $76.5 \%$ vs. $83.9 \%, p$-value 0.004). No differences in non-surgical complications could be observed, indicating the differences in morbidity are caused by perioperative complications like wound dehiscence, anastomotic insufficiency or ileus, as reported in other studies [23-25]. One major factor leading to reduced overall survival in patients with postoperative complications is the reduced ability to receive adjuvant treatment [26-28]. Interestingly, we did not see any differences in adjuvant treatment rates between the BMI-groups, which leaves the mechanism behind the observed differences in the overall survival unanswered.

\subsection{Local Recurrence (LR)}

Tumor localization in the rectum is known to affect the risk for the development of LR after primary curative surgical resection in rectal cancer patients [29]. Reduced surgical visibility in obese patients with lower rectal tumors compromising sufficient resection, are possible responsible factors. Despite these findings, patients included in our study did not show significantly higher rates of LR dependent on tumor localization, which might be correlated to the surgical procedure. APE has been proven to be a significant risk factor for LR in patients with rectal carcinoma [30]. Our patient cohort did not show the same dependency. Localization in the lower rectum or advanced tumor stage are common reasons to perform APE. While anatomy and tumor localization may be reasons for increased recurrences, tumor biology is another explanation worth considering. Obesity is associated with insulin resistance and consequently higher rates of circulating insulin [31] leading to a higher bioactivity of Insulin-like-growth factor 1 (IGF-1). IGF-1 promotes a series of intracellular signaling cascades leading to mitogenic and antiapoptotic events, a risk factor for recurrence and tumor growth [32,33]. No relation was seen between LR and tumor localization, whereas an advanced tumor stage increased LR risk.

\subsection{Distant Metastasis (DM)}

We were able to show a correlation of DM-rates by BMI and the UICC-stage. Our study population displayed increased rates of DM in patients with high or very low BMI $\left(<18.5 \mathrm{or} \geq 35 \mathrm{~kg} / \mathrm{m}^{2}\right)$. The disparate appearance of DM might be due to molecular mechanisms as suggested by different studies pointing on inflammatory cytokines such as insulin-like growth factor-receptor (IGF-R) and leptin [34-37]. With obesity inducing a state of slight inflammation and changing the microenvironment for example in steatotic livers, this might be a reason for higher rates of DM in very obese patients [38]. Unfortunately, we have not been able to confirm this hypothesis due to missing blood samples for the patients included within this analysis. In underweight patients instead, the earlier infiltration of blood and lymph vessels can be seen as a factor for higher DM-rates.

The possibility to provide adjuvant tumor therapy for patients with DM was diverging due to BMI (Group 1 vs. Group 2: $69.8 \%$ vs. $60.0 \%$, $p$-value 0.036 ). This may be an effect of comorbidities in patients with high and very low BMI. 


\subsection{Overall Survival}

Patients with advanced tumors are known to have a decreased time of overall survival [39]. This circumstance is reflective of the advanced stage of the tumor-disease.

The results of our study suggest an association between BMI and overall survival and are consistent with a study performed on 526 patients, determining a link between BMI and patients outcome diagnosed with left-sided cancer and rectal cancer [40]. Reduced survival in patients with obesity class II or higher as well as underweight (Group 2) compared to normal weight, overweight and obesity class I (Group 1) individuals, may be related to higher rates of distant metastases (DM) found in this group. At least the lower survival rate in patients with underweight might be derived from an advanced tumor stage causing a catabolic metabolism. Weight-loss promoted by this catabolic situation could be a factor shifting patients with normal weight to underweight at the time of hospital admission [9]. Chemotherapy-related toxicities and other complications had been observed in slightly lower numbers in obese patients compared to non-obese patients in some adjuvant therapy trials [14,41]. The possibility to provide appropriate postoperative tumor therapy can be suggested as a factor for better survival in obese patients.

Tumor localization in contrast to other trials, had no significant impact on the overall survival of patients included in this study [42]. This may be reflective of a good surgical quality independent of difficult conditions.

The strength of our study is the large sample size, stage specific data and the long-term follow-up data with at least 5-years follow up-time for each patient, as well as completeness of the data. On the other hand, this study has certain limitations: we performed a retrospective analysis of prospectively collected data. The small number of patients in the subgroups as well as the design as a single center study reduced the validity of our conclusions.

\section{Materials and Methods}

The initially screened study cohort consisted of patients diagnosed with rectal cancer at the University Hospital Erlangen in the time of January 2003 until December 2010. All of these patients underwent surgery for the treatment of rectal carcinoma in the department of surgery at the University Hospital Erlangen. Patient data were selected on the following inclusion criteria: Solitary invasive rectal carcinoma (at least infiltration of the submucosa) $\leq 16 \mathrm{~cm}$ from anal verge when measured with a rigid sigmoidorectoscope; treated by (low) anterior resection, Hartmann procedure, intersphincteric resection or abdominoperineal excision; no other malignant tumors either synchronous with or prior to diagnosis; no history of familial adenomatous polyposis, ulcerative colitis or Crohn's disease; no distant metastasis (M0) at the time of diagnosis. 6 patients without valid BMI, 6 patients who died postoperatively and three patients with unknown tumor-status were excluded from the analysis.

Only patients with microscopic curative resection (R0) were used for outcome analyses. Consequently, a total of 598 patients were included for these analyses.

To calculate surgical quality indicators like R0-resection rate in Table 3 we included all patients independent of their resection status $(\mathrm{R} 0(n=598)+\mathrm{R} 1(n=9)+\mathrm{R} 2(n=5)=612)$.

Long-term chemoradiation was administered in the majority of patients after preoperative staging (cT3, 4 or $\mathrm{cN}+$ [43]), in concordance to the protocol of the German CAO/ARO/AIO-94 study [44]. The neoadjuvant treatment was finished 6-8 weeks prior to surgery.

All samples were categorized according to the seventh edition of the UICC TNM classification [45].

According to the definition of the World Health Organization (WHO) the patient cohort was divided in six groups, i.e., underweight (BMI $<18.5 \mathrm{~kg} / \mathrm{m}^{2} ; n=5$ ); normal weight (BMI 18.5-24,9 kg/ $\mathrm{m}^{2}$; $n=209$ ); overweight (BMI $25.0-29.9 \mathrm{~kg} / \mathrm{m}^{2} ; n=257$ ); obesity class I (BMI $30.0-34.9 \mathrm{~kg} / \mathrm{m}^{2} ; n=102$ ); obesity class II (BMI $35.0-39.9 \mathrm{~kg} / \mathrm{m}^{2} ; n=16$ ); and obesity class III (BMI $\geq 40.0 \mathrm{~kg} / \mathrm{m}^{2} ; n=9$ ) [46]. BMI was measured in the anaesthesia outpatient clinic during preparation for surgery at the time of first hospital admission at the University Hospital Erlangen. 
To meet the challenge of a society with an increasing prevalence of obesity in the last 30 years [47], we divided the study population in two groups (Group 1 BMI 18.5- $35 \mathrm{~kg} / \mathrm{m}^{2} ; n=568$; Group 2 BMI $<18.5$ and $\geq 35 \mathrm{~kg} / \mathrm{m}^{2} ; n=30$ ) with Group 1 representing the dominant phenotype in future communities.

Patients with tumors in the lower and middle rectum received a total mesorectal excision (TME). Partial mesorectal excision (PME) or TME was performed if suitable in patients with tumour in the upper part of the rectum. The quality of TME or PME was examined in accordance to the protocol of Quirke et al. by a pathologist [48].

All patients were followed up with for at least 5 years: twice a year, for the first 2 years and then yearly for the remaining period as suggested by the first edition of the German S3-Guidelines for Colorectal Carcinoma [43]. Follow-ups included physical examination, analysis of carcinoembryonic antigen (CEA) levels, chest X-ray, abdominal ultrasonography, computed tomography of the pelvis and rectoscopy.

Follow-up data were either collected by standard follow-up examinations at the University hospital Erlangen or by written correspondence with the patient's general practitioner. Subsequently the vital status of the patient was validated through inquiries at the patient's local registration office and all data have been collected and categorized by our local cancer registry.

All subjects gave their informed consent for inclusion before they participated in this study. The study was conducted in accordance with the Declaration of Helsinki, and the protocol was approved by the Ethics Committee of the Friedrich-Alexander-Universität Erlangen-Nürnberg, Germany (172_19 Bc).

\section{Statistical Analysis}

To compare categorical data $\chi^{2}$ and Fisher's exact test were used. For quantitative data the Mann-Whitney $U$ test was applied. The Kaplan-Meier method was utilized to analyze the five-year rates of local recurrence, distant metastasis and overall survival; the log-rank test was applied for comparison of the survival curves. For overall survival death from any cause was defined as an event. To analyze univariable and multivariable differences in local recurrence, distant metastasis or overall survival, a Cox-regression model was used. A $p$-value $<0,050$ was considered to be significant.

The statistical software package SPSS ${ }^{\circledR}$ version 24 (IBM, Armonk, NY, USA) was used for all analyses.

\section{Conclusions}

We were able to show differences in survival rates depending on BMI values. The increased rate of distant metastases as well as the higher rates of APE in Group 2 might be due to patient selection in our cohort. Patients treated by APE had a 2-fold risk of death, but no elevated rates of DM. LR increased the rate of DM by a factor of 3,6, but showed no impact on overall survival.

Author Contributions: Conceptualization, M.K. and G.F.W.; methodology, M.K., G.F.W.; software, M.K.; validation, M.K., S.M., G.F.W.; formal analysis, M.K.; investigation, M.K., G.F.W.; resources, S.M.; data curation, M.K., M.C.L., S.M., and G.F.W.; writing—original draft preparation, M.K.; writing—review and editing, M.K., M.C.L., S.M., C.K., M.B., A.B., C.P., R.G., G.F.W.; visualization, M.K. and G.F.W.; operation: K.W; supervision, G.F.W.; project administration, G.F.W.; funding acquisition, G.F.W.

Funding: This research was supported by the german research foundation (DFG) to G.F.W. (WE 4892/4-1).

Acknowledgments: The present work was performed in fulfilment of the requirements for obtaining the degree "med." at the Friedrich-Alexander-Universität Erlangen-Nürnberg (FAU).

Conflicts of Interest: The authors declare no conflict of interest.

\section{References}

1. Krebs-Datenbankabfrage. Available online: https://www.krebsdaten.de/Krebs/SiteGlobals/Forms/ Datenbankabfrage/datenbankabfrage_stufe2_form.html (accessed on 15 December 2017).

2. Brenner, H.; Stegmaier, C.; Ziegler, H. Long-term survival of cancer patients in Germany achieved by the beginning of the third millenium. Ann. Oncol. 2005, 16, 981-986. [CrossRef] 
3. Luengo-Fernandez, R.; Leal, J.; Gray, A.; Sullivan, R. Economic burden of cancer across the European Union: A population-based cost analysis. Lancet Oncol. 2013, 14, 1165-1174. [CrossRef]

4. Deng, T.; Lyon, C.J.; Bergin, S.; Caligiuri, M.A.; Hsueh, W.A. Obesity, Inflammation, and Cancer. Annu. Rev. Pathol. 2016, 11, 421-449. [CrossRef]

5. Ma, Y.; Yang, Y.; Wang, F.; Zhang, P.; Shi, C.; Zou, Y.; Qin, H. Obesity and risk of colorectal cancer: A systematic review of prospective studies. PLoS ONE 2013, 8, e53916. [CrossRef]

6. Hoda, M.R.; Keely, S.J.; Bertelsen, L.S.; Junger, W.G.; Dharmasena, D.; Barrett, K.E. Leptin acts as a mitogenic and antiapoptotic factor for colonic cancer cells. Br. J. Surg. 2007, 94, 346-354. [CrossRef] [PubMed]

7. Giovannucci, E. Physical Activity, Obesity, and Risk for Colon Cancer and Adenoma in Men. Ann. Intern. Med. 1995, 122, 327-334. [CrossRef]

8. Moghaddam, A.A.; Woodward, M.; Huxley, R. Obesity and Risk of Colorectal Cancer: A Meta-analysis of 31 Studies with 70,000 Events. Cancer Epidemiol. Prev. Biomark. 2007, 16, 2533-2547. [CrossRef]

9. Gebauer, B.; Meyer, F.; Ptok, H.; Steinert, R.; Otto, R.; Lippert, H.; Gastinger, I. Impact of Body Mass Index on Early Postoperative and Long-Term Outcome after Rectal Cancer Surgery. Visc. Med. 2017, 33, 373-382. [CrossRef] [PubMed]

10. Klil-Drori, A.J.; Azoulay, L.; Pollak, M.N. Cancer, obesity, diabetes, and antidiabetic drugs: Is the fog clearing? Nat. Rev. Clin. Oncol. 2017, 14, 85-99. [CrossRef]

11. Larsson, S.C.; Wolk, A. Obesity and colon and rectal cancer risk: A meta-analysis of prospective studies. Am. J. Clin. Nutr. 2007, 86, 556-565. [CrossRef]

12. Bianchini, F.; Kaaks, R.; Vainio, H. Overweight, obesity, and cancer risk. Lancet Oncol. 2002, 3, 565-574. [CrossRef]

13. Lee, J.; Meyerhardt, J.A.; Giovannucci, E.; Jeon, J.Y. Association between body mass index and prognosis of colorectal cancer: A meta-analysis of prospective cohort studies. PLoS ONE 2015, 10, e0120706. [CrossRef]

14. Meyerhardt, J.A.; Tepper, J.E.; Niedzwiecki, D.; Hollis, D.R.; McCollum, A.D.; Brady, D.; O'Connell, M.J.; Mayer, R.J.; Cummings, B.; Willett, C.; et al. Impact of Body Mass Index on Outcomes and Treatment-Related Toxicity in Patients With Stage II and III Rectal Cancer: Findings From Intergroup Trial 0114. J. Clin. Oncol. 2004, 22, 648-657. [CrossRef] [PubMed]

15. Campbell, P.T.; Newton, C.C.; Dehal, A.N.; Jacobs, E.J.; Patel, A.V.; Gapstur, S.M. Impact of Body Mass Index on Survival After Colorectal Cancer Diagnosis: The Cancer Prevention Study-II Nutrition Cohort. J. Clin. Oncol. 2012, 30, 42-52. [CrossRef] [PubMed]

16. Fedirko, V.; Romieu, I.; Aleksandrova, K.; Pischon, T.; Trichopoulos, D.; Peeters, P.H.; Romaguera-Bosch, D.; Bueno-de-Mesquita, H.B.; Dahm, C.C.; Overvad, K.; et al. Pre-diagnostic anthropometry and survival after colorectal cancer diagnosis in Western European populations. Int. J. Cancer 2014, 135, 1949-1960. [CrossRef]

17. Schroen, A.T.; Cress, R.D. Use of Surgical Procedures and Adjuvant Therapy in Rectal Cancer Treatment. Ann. Surg. 2001, 234, 641-651. [CrossRef] [PubMed]

18. Ciga Lozano, M.Á.; Codina Cazador, A.; Ortiz Hurtado, H. Cancer Project Recto of the Spanish Association of Surgeons Oncological results according to type of resection for rectal cancer. Cir. Esp. 2015, 93, 229-235. [CrossRef]

19. Warschkow, R.; Ebinger, S.M.; Brunner, W.; Schmied, B.M.; Marti, L. Survival after Abdominoperineal and Sphincter-Preserving Resection in Nonmetastatic Rectal Cancer: A Population-Based Time-Trend and Propensity Score-Matched SEER Analysis. Gastroenterol. Res. Pract. 2017, 2017. [CrossRef]

20. de Melo, G.M.; Ribeiro, K.C.; Kowalski, L.P.; Deheinzelin, D. Risk factors for postoperative complications in oral cancer and their prognostic implications. Arch. Otolaryngol. Head Neck Surg. 2001, 127, 828-833.

21. Rizk, N.P.; Bach, P.B.; Schrag, D.; Bains, M.S.; Turnbull, A.D.; Karpeh, M.; Brennan, M.F.; Rusch, V.W. The impact of complications on outcomes after resection for esophageal and gastroesophageal junction carcinoma. J. Am. Coll. Surg. 2004, 198, 42-50. [CrossRef] [PubMed]

22. Law, W.L.; Choi, H.K.; Lee, Y.M.; Ho, J.W. The impact of postoperative complications on long-term outcomes following curative resection for colorectal cancer. Ann. Surg. Oncol. 2007, 14, 2559-2566. [CrossRef]

23. Hrabe, J.E.; Sherman, S.K.; Charlton, M.E.; Cromwell, J.W.; Byrn, J.C. Effect of BMI on outcomes in proctectomy. Dis. Colon Rectum 2014, 57, 608-615. [CrossRef]

24. Bokey, L.; Chapuis, P.H.; Dent, O.F. Impact of obesity on complications after resection for rectal cancer. Colorectal Dis. 2014, 16, 896-906. [CrossRef] 
25. Tjeertes, E.E.K.M.; Hoeks, S.S.E.; Beks, S.S.B.J.C.; Valentijn, T.T.M.; Hoofwijk, A.A.G.M.; Stolker, R.J.R.J. Obesity-A risk factor for postoperative complications in general surgery? BMC Anesthesiol. 2015, 15, 112. [CrossRef] [PubMed]

26. Merkow, R.P.; Bentrem, D.J.; Mulcahy, M.F.; Chung, J.W.; Abbott, D.E.; Kmiecik, T.E.; Stewart, A.K.; Winchester, D.P.; Ko, C.Y.; Bilimoria, K.Y. Effect of postoperative complications on adjuvant chemotherapy use for stage III colon cancer. Ann. Surg. 2013, 258, 847-853. [CrossRef] [PubMed]

27. Jin, L.X.; Sanford, D.E.; Squires, M.H.; Moses, L.E.; Yan, Y.; Poultsides, G.A.; Votanopoulos, K.I.; Weber, S.M.; Bloomston, M.; Pawlik, T.M.; et al. Interaction of Postoperative Morbidity and Receipt of Adjuvant Therapy on Long-Term Survival After Resection for Gastric Adenocarcinoma: Results From the U.S. Gastric Cancer Collaborative. Ann. Surg. Oncol. 2016, 23, 2398-2408. [CrossRef]

28. Donat, S.M.; Shabsigh, A.; Savage, C.; Cronin, A.M.; Bochner, B.H.; Dalbagni, G.; Herr, H.W.; Milowsky, M.I. Potential Impact of Postoperative Early Complications on the Timing of Adjuvant Chemotherapy in Patients Undergoing Radical Cystectomy: A High-Volume Tertiary Cancer Center Experience. Eur. Urol. 2009, 55, 177-186. [CrossRef] [PubMed]

29. Stipa, S.; Nicolanti, V.; Botti, C.; Cosimelli, M.; Mannella, E.; Stipa, F.; Giannarelli, D.; Bangrazi, C.; Cavaliere, R. Local recurrence after curative resection for colorectal cancer: Frequency, risk factors and treatment. J. Surg. Oncol. Suppl. 1991, 2, 155-160. [CrossRef]

30. den Dulk, M.; Putter, H.; Collette, L.; Marijnen, C.A.M.; Folkesson, J.; Bosset, J.-F.; Rödel, C.; Bujko, K.; Påhlman, L.; van de Velde, C.J.H. The abdominoperineal resection itself is associated with an adverse outcome: The European experience based on a pooled analysis of five European randomised clinical trials on rectal cancer. Eur. J. Cancer 2009, 45, 1175-1183. [CrossRef]

31. Kissebah, A.H.; Vydelingum, N.; Murray, R.; Evans, D.J.; Hartz, A.J.; Kalkhoff, R.K.; Adams, P.W. Relation of body fat distribution to metabolic complications of obesity. J. Clin. Endocrinol. Metab. 1982, 54, 254-260. [CrossRef]

32. Valentinis, B.; Baserga, R. IGF-I receptor signalling in transformation and differentiation. Mol. Pathol. 2001, 54, 133-137. [CrossRef]

33. Yu, H.; Rohan, T. Role of the insulin-like growth factor family in cancer development and progression. J. Natl. Cancer Inst. 2000, 92, 1472-1489. [CrossRef]

34. Wang, Z.; Aguilar, E.G.; Luna, J.I.; Dunai, C.; Khuat, L.T.; Le, C.T.; Mirsoian, A.; Minnar, C.M.; Stoffel, K.M.; Sturgill, I.R.; et al. Paradoxical effects of obesity on T cell function during tumor progression and PD-1 checkpoint blockade. Nat. Med. 2019, 25, 141-151. [CrossRef]

35. Clements, V.K.; Long, T.; Long, R.; Figley, C.; Smith, D.M.C.; Ostrand-Rosenberg, S. Frontline Science: High fat diet and leptin promote tumor progression by inducing myeloid-derived suppressor cells. J. Leukoc. Biol. 2018, 103, 395-407. [CrossRef]

36. Hakam, A.; Yeatman, T.J.; Lu, L.; Mora, L.; Marcet, G.; Nicosia, S.V.; Karl, R.C.; Coppola, D. Expression of insulin-like growth factor-1 receptor in human colorectal cancer. Hum. Pathol. 1999, 30, 1128-1133. [CrossRef]

37. Wu, Y.; Yakar, S.; Zhao, L.; Hennighausen, L.; LeRoith, D. Circulating Insulin-like Growth Factor-I Levels Regulate Colon Cancer Growth and Metastasis. Cancer Res. 2002, 62, 1030-1035.

38. Wu, Y.; Brodt, P.; Sun, H.; Mejia, W.; Novosyadlyy, R.; Nunez, N.; Chen, X.; Mendoza, A.; Hong, S.-H.; Khanna, C.; et al. Insulin-Like Growth Factor-I Regulates the Liver Microenvironment in Obese Mice and Promotes Liver Metastasis. Cancer Res. 2010, 70, 57-67. [CrossRef] [PubMed]

39. Compton, C.C.; Greene, F.L. The Staging of Colorectal Cancer: 2004 and Beyond. CA Cancer J. Clin. 2004, 54, 295-308. [CrossRef]

40. Haydon, A.M.M.; MacInnis, R.J.; English, D.R.; Giles, G.G. Effect of physical activity and body size on survival after diagnosis with colorectal cancer. Gut 2006, 55, 62-67. [CrossRef]

41. Meyerhardt, J.A.; Catalano, P.J.; Haller, D.G.; Mayer, R.J.; Benson, A.B.; Macdonald, J.S.; Fuchs, C.S. Influence of body mass index on outcomes and treatment-related toxicity in patients with colon carcinoma. Cancer 2003, 98, 484-495. [CrossRef] [PubMed]

42. Das, P.; Skibber, J.; Rodriguez-Bigas, M.; Feig, B.; Chang, G.; Hoff, P.; Eng, C.; Wolff, R.; JanJan, N.; Delclos, M.; et al. Clinical and Pathologic Predictors of Locoregional Recurrence, Distant Metastasis, and Overall Survival in Patients Treated with Chemoradiation and Mesorectal Excision for Rectal Cancer. Am. J. Clin. Oncol. 2006, 29, 219-224. [CrossRef] 
43. Pox, C.; Aretz, S.; Bischoff, S.; Graeven, U.; Hass, M.; Heußner, P.; Hohenberger, W.; Holstege, A.; Hübner, J.; Kolligs, F.; et al. S3-Leitlinie Kolorektales Karzinom, Kurzversion 1.1, AWMF Registrierungsnummer: 021/007OL; Leitlinienprogramm Onkologie: Berlin, Germany, 2014.

44. Sauer, R.; Becker, H.; Hohenberger, W.; Rödel, C.; Wittekind, C.; Fietkau, R.; Martus, P.; Tschmelitsch, J.; Hager, E.; Hess, C.F.; et al. Preoperative vs. Postoperative Chemoradiotherapy for Rectal Cancer. N. Engl. J. Med. 2004, 351, 1731-1740. [CrossRef]

45. Sobin, L.; Gospodarowicz, M.; Wittekind, C. TNM Classification of Malignant Tumours, 7th ed.; Available online: https://www.wiley.com/en-us/TNM+Classification+of+Malignant+Tumours\%2C+7th+Edition-p9781444358964 (accessed on 12 August 2018).

46. World Health Organization (WHO). Global Database on Body Mass Index. Available online: http://apps. who.int/bmi/index.jsp?introPage=intro_3.html (accessed on 12 August 2018).

47. NCD Risk Factor Collaboration (NCD-RisC). Trends in adult body-mass index in 200 countries from 1975 to 2014: A pooled analysis of 1698 population-based measurement studies with $19 \cdot 2$ million participants. Lancet 2016, 387, 1377-1396. [CrossRef]

48. Quirke, P.; Durdey, P.; Dixon, M.F.; Williams, N.S. Local recurrence of rectal adenocarcinoma due to inadequate surgical resection. Histopathological study of lateral tumour spread and surgical excision. Lancet 1986, 2, 996-999. [CrossRef]

(C) 2019 by the authors. Licensee MDPI, Basel, Switzerland. This article is an open access article distributed under the terms and conditions of the Creative Commons Attribution (CC BY) license (http://creativecommons.org/licenses/by/4.0/). 